\title{
CAPITAL SOCIAL, REDES SOCIALES Y ACTITUD EMPRENDEDORA EN ESTUDIANTES UNIVERSITARIOS DE REPÚBLICA DOMINICANA
}

\section{Social capital, social networks and entrepreneurial attitude of university students from Dominican Republic}

\author{
José Feliz Marrero ${ }^{1}$
}

Recibido: 11-11-2016• Aprobado: 16-2-2017

\section{Resumen}

El objetivo del presente estudio es conocer en qué medida el capital social y las redes sociales influyen en el desarrollo de la actitud emprendedora de los estudiantes, para lo cual se tomó una muestra de 92 sujetos que estudian diversas carreras y programas en cinco universidades de República Dominicana. Los hallazgos encontrados indican que los factores sociodemográficos de influencia no afectan la actitud emprendedora de los estudiantes y, por lo tanto, no existen diferencias significativas entre ellos con respecto a su comportamiento emprendedor de manera individual y entre las universidades. En cambio, los factores psicológicos como la necesidad de logros y la propensión al riesgo, así como los factores sociológicos analizados, tales como el capital social, las redes sociales y el entorno familiar tienen una relación significativa con la actitud emprendedora de los individuos, considerándose los de mayor influencia en el desarrollo del comportamiento emprendedor. Por otro lado, no se evidenciaron diferencias significativas entre estas características del perfil emprendedor por universidades, comprobando que los elementos predominantes en la determinación del comportamiento emprendedor están más ligados a factores personales y de ascendencia familiar. Estos resultados confirman la teoría del aprendizaje social que defiende la idea de que los individuos prestan atención a los modelos de referencias, por sus aportes al aprendizaje de nuevas competencias, tareas, habilidades y normas que le dan sentido a su contexto; asimismo, se comprueba la teoría de aprendizaje indirecto que explica la manera en que los modelos de referencia influyen en el comportamiento de las personas.

Palabras clave: capital social; redes sociales; actitud emprendedora; reciprocidad; entorno familiar; República Dominicana.

1. Profesor del INTEC, República Dominicana.

Correo electrónico: jose.feliz@intec.edu.do

\section{Abstract}

The objective of this research is to know the extent to which social capital and social networks influence the development of student entrepreneurship in a sample of 92 subjects who study different careers and programs at five universities in the Dominican Republic. It is a non-probabilistic study, with an intentionally selected sample represented by 92 students of five universities in the country: UNAPEC, INTEC, PUCMM, UNIBE and UNPHU that have had initiatives and entrepreneurship projects. Findings indicate that socio-demographic influencing factors do not affect students' entrepreneurial attitude and therefore there are no significant differences between them regarding their entrepreneurial behavior individually and between universities. On the other hand, the psychological factors: need for achievement and risk propensity and the sociological ones analyzed: social capital, social networks and family environment have a significant relationship with the entrepreneurial attitude of individuals. Being considered those of greater influence in the development of entrepreneurial behavior. According to the results, there were no significant differences between these characteristics of the entrepreneurial profile by universities, proving that the predominant elements in the determination of entrepreneurial behavior are more linked to personal factors and family ancestry. These results confirm the theory of social learning that defends the idea that individuals pay attention to reference models, for their contributions to learning new competencies, tasks, skills and norms that give meaning to their context and the theory of indirect learning that explains how reference models influence people's behavior.

Keywords: social capital; social networks; entrepreneurial attitude; reciprocity; family environment; Dominican Republic. 


\section{Introducción}

En la actualidad existe un interés particular por el desarrollo del capital social y la influencia del uso de las redes sociales, así como por promover en la sociedad y, principalmente, en el ámbito académico el desarrollo de una actitud emprendedora, esto debido a la importancia y a los efectos positivos del emprendedurismo en el desarrollo económico y social.

Algunos autores consideran que el capital social es productivo y posibilita el logro de ciertos fines que no se alcanzarían en su ausencia (Putman, 1995; Coleman, 1998 \& Bourdieu, 1986). Otros autores como Baron (2000) expresan que, aunque las características del emprendedor son muy importantes, los factores externos son determinantes en la decisión de crear una empresa.

Autores tales como Veciana \& Urbano (2005) afirman que el estudio de las intenciones emprendedoras es un campo del que aún falta mucho por investigar, por lo que resulta de especial interés introducirse en el estudio de las intenciones emprendedoras desde el ámbito académico.

El objetivo de este trabajo es aportar evidencia empírica sobre la relación del capital social y las redes sociales con el desarrollo de la actitud emprendedora de los estudiantes universitarios de Santo Domingo. La población objeto de estudio alcanzó los estudiantes emprendedores de cinco universidades del país: INTEC, PUCMM, UNIBE, UNAPEC y UNPHU, por ser las que tienen programas y estructuras formales de fomento al emprendedurismo.

La investigación se focalizó en el análisis de tres grupos de variables: sociodemográficas, psicológicas y sociológicas relacionales. Los datos se obtuvieron de 92 estudiantes emprendedores que respondieron el cuestionario. Para los fines de este estudio se define la actitud emprendedora como la predisposición que se tiene hacia un comportamiento emprendedor, que implica las características del ser humano y del medio en el que opera, lo que representa, en sentido amplio, un conducto dedicado al hombre mismo, a sus acciones, sus visiones del mundo y la manera que tiene de transformar la realidad.

Se infiere que si se mide la actitud es posible predecir cuál será el comportamiento del individuo (Ajzen, 2001). Es evidente que las actitudes evalúan sentimientos, acciones, decisiones y, por tanto, son elementos importantes en la explicación de la conducta humana.

Las cuestiones fundamentales a responder son: ¿cuáles son los factores sociodemográficos, psicológicos y sociológicos que fomentan el desarrollo de la actitud emprendedora de los estudiantes universitarios en Santo Domingo? ¿Cuál es la relación entre la necesidad de logro y la propensión al riesgo con la actitud emprendedora de los estudiantes?; ¿cuál es la relación del capital social con la actitud emprendedora?; el uso de las redes sociales influye en la actitud emprendedora del estudiante universitario?; ¡las normas subjetivas que representan el entorno del estudiante (familia, compañeros de estudio y profesores) influyen en la intención emprendedora del estudiante universitario?

El trabajo se ha estructurado de la siguiente forma: en primer lugar, el planteamiento del problema de investigación, el marco teórico, la revisión de la literatura empírica previa y las hipótesis a contrastar; en segundo lugar, se expone la metodología y se define un marco conceptual donde se identifican las variables a estudiar; en tercer lugar, se lleva a cabo el análisis de los resultados y, finalmente, se presentan las principales conclusiones alcanzadas.

Los factores que se analizaron fueron los siguientes: sociodemográfico, psicológico y sociológico. Los factores sociodemográficos estudiados fueron la edad, el género y el nivel educativo. Mientras que los factores psicológicos examinados fueron la necesidad de logros y la propensión al riesgo. Dentro 
de los factores sociológicos o relacionales que afectan la actitud emprendedora se estudió el capital social, las redes sociales y el entorno familiar.

\section{Marco teórico y revisión de estudios previos}

Ahora trataremos de responder la siguiente interrogante:

¿Los factores sociodemográficos, psicológicos y sociológicos incluyen o no la actitud emprendedora?

En algunos estudios se evidencia una relación positiva entre las variables sociodemográficas como la edad, el género, el nivel educativo y el capital humano con la intención de los individuos de emprender nuevos negocios. En el caso de la edad, Levesque \& Minniti (2006) consideran que existe una relación inversa entre dicha variable y la probabilidad de acometer la puesta en marcha de un nuevo negocio.

En el caso de la variable género, algunos estudios concluyen que hay pocas diferencias en las características personales entre hombres y mujeres que inician una actividad empresarial; sin embargo, otros autores entienden que cada vez existe más literatura especializada que demuestra una mayor preferencia de los varones hacia la creación de empresas (Veciana et al., 2005). De acuerdo con lo anterior, se advierte que no existen criterios unificados con respecto a la influencia de esta variable en la actitud emprendedora de las personas.

Igual comportamiento evidencia la variable nivel educativo con relación a la actitud emprendedora. De acuerdo con Blanchflower (2004), la relación entre emprendimiento y educación es positivamente significativa. Estas conclusiones contraponen los resultados de estudios realizados por Prahalad \& Bettis (1986), que sostienen la idea de que el nivel educativo puede limitar la actitud emprendedora de las personas, debido a que un mayor conocimiento del individuo incrementará los límites para la identificación de oportunidades, pudiendo generar una miopía de mercado, limitando de esta manera la actitud emprendedora de los individuos.

Teniendo como base estos argumentos, se propone la siguiente hipótesis de trabajo:

H1 Hay una relación significativa entre las variables sociodemográficas: edad, género y nivel educativo, con la actitud emprendedora.

\subsection{Factores sociológicos o relacionales}

La propensión hacia el riesgo y la necesidad de logro son características psicológicas comunes que determinan el comportamiento emprendedor. Según McClelland (1961), citado por Graña (2002), la "oferta" de emprendedores está explicada principalmente por la existencia de motivación, siendo el disparador principal de la misma la necesidad de logro que tiene el emprendedor. Esta última es concebida como el deseo que tienen las personas de ser exitosas (Robbins, 2011), así como el impulso de sobresalir, de tener logros con relación a un conjunto de normas y de luchar por lograr sus metas.

De modo que la oferta de emprendedores depende de la necesidad psicológica de logro del individuo, en lugar del deseo de poseer dinero, aun cuando la retribución monetaria puede constituir un símbolo de logro del emprendedor (Grańa, 2002). Este rasgo de los seres humanos de querer lograr cada vez más los enfrenta a situaciones contingentes y riesgosas, induciendo a una atención particular del contexto en el que se encuentra, siendo esta situación determinada en gran medida por la propensión al riesgo que cada individuo posee (Catalso et al., 2012). Esta debe ser una característica fundamental de todo empresario (Cano et al., 2004).

Al investigar la necesidad de realización, McClelland (1961), citado por Robbins y Judge (2011), encontró que los grandes realizadores se diferencian 
de otras personas por su deseo de hacer mejor las cosas. La necesidad de logro, como rasgo de la personalidad, tiene una relación positiva sobre la intención emprendedora (Entrialgo et al., 1999).

Esta argumentación proporciona el fundamento para las siguientes hipótesis:

H2a. La propensión al riesgo tiene una relación positiva con la actitud emprendedora de los estudiantes universitarios.

H2b. La necesidad de logro se relaciona positivamente con la actitud emprendedora de los estudiantes universitarios.

\subsection{Factores sociológicos o relacionales}

De acuerdo con Graña (2002), el enfoque sociológico relaciona la actividad de creación de una empresa con un conjunto de factores culturales, económicos, sociológicos y psicológicos, donde las características son adquiridas a través de la vida del individuo en su proceso de socialización. Gibb \& Ritchie (1982) explican que al analizar las características de los emprendedores es inevitable incorporar los factores sociológicos o relacionales como determinantes de la actitud emprendedora, tales como el capital social, las redes de contactos y el entorno familiar.

\subsubsection{Capital social: conceptualizaciones}

En el contexto académico, el concepto de capital social ha ido convirtiéndose en un importante factor predominante para la sociedad y el desarrollo del territorio, lo que ha generado amplios debates en este espacio de construcción del conocimiento. Entre los autores que se enmarcan dentro de estas controversias conceptuales podemos nombrar a Bourdieu (1986), Coleman (1990), Putnam (1993), Burt (2000), entre otros.

Una de las primeras definiciones sistemáticas y contemporáneas de capital social que puede hallarse es la que presenta Bourdie (1985), que lo define como "el agregado de los recursos reales o potenciales que se vinculan con la posesión de una red duradera de relaciones más o menos institucionalizadas de conocimiento o reconocimiento mutuo" (Bourdieu, 1985, p. 248, citado por Portes, 1999).

En ese mismo sentido, según Coleman (1990), el capital social constituye un recurso cuya particularidad radica en ser algo inherente a la estructura de las relaciones sociales. Este autor entiende que la existencia de redes densas es una condición necesaria para que emerja el capital social, en tanto el aumento de escala en las relaciones sociales estables pasa de un contrato diádico entre dos individuos a redes ego-centradas, de las cuales pueden emerger instituciones comunitarias de capital social (Durston, 2000).

Según el Banco Mundial, el capital social está vinculado a las "normas de confianza", los "valores", las "actitudes y redes" existentes entre individuos e instituciones, como parte de la "asociación" o las "acciones colectivas y la cooperación" (Banco Mundial, 1999).

En palabras de Rangel (2008), el grado de confianza y asociación son las dos variables claves en la formación del capital social de una comunidad. Este autor afirma que cuanto mayor es la confianza en las organizaciones, mayor será el capital social. Es por ello que la confianza es una dimensión crítica del capital social.

Los recursos asociativos que importan para dimensionar el capital social de un entorno productivo son las relaciones de confianza, reciprocidad y cooperación (Astri, 2001). Asimismo, Putnam (2002) advierte que el capital social promueve la cooperación, ya que la estructura de la interacción requiere reciprocidad e intercambio de clientes potenciales. En la misma línea, Montesinos et al. (2005) afirma que los empresarios no están aislados, sino llamados a interactuar a través de relaciones de reciprocidad, solidaridad y confianza. 
Desde la perspectiva de la teoría de la acción racional, Coleman (1998) considera que la confianza es el sustento de las relaciones de reciprocidad en que se basa el capital social, de ahí el carácter que reviste esta dimensión como recurso inmerso en las relaciones sociales, del que se pueden obtener beneficios individuales.

Astri (2001) plantea que el capital social de un emprendedor se define por las relaciones de confianza, reciprocidad y cooperación. De modo similar, en el enfoque interactivo, Coleman (1990) introduce la posibilidad de considerar las relaciones de cooperación y trabajo en red como una forma del capital, ya que se pueden acumular, almacenar y convertir en otras especies apropiadas. La teoría de redes ha dado lugar a una corriente de investigaciones alrededor del impacto de la red (network) en el proceso de creación de empresas (Johannisson, 2006).

El análisis de una red social parte de la identificación de su estructura, compuesta por el tipo de relaciones y acciones sociales que existen entre sus miembros y cómo estas se manifiestan a través de conductas individuales interconectadas, dando paso a la conducta del colectivo; en otras palabras, al macro-comportamiento de la red.

Tomando en cuenta los aspectos anteriores, se proponen las siguientes hipótesis de trabajo:

- Hipótesis 3a. El desarrollo del capital social se relaciona positivamente con el desarrollo de la actitud emprendedora de los estudiantes.

- Hipótesis 3b. Hay relación significativa entre reciprocidad y actitud emprendedora.

- Hipótesis 4. El nivel de confianza percibida por los estudiantes emprendedores está pasivamente relacionado con la actitud emprendedora.

- Hipótesis 5. Hay relación significativa entre las redes sociales y la actitud emprendedora del estudiante universitario.

\subsubsection{Entorno familiar}

Para Scout \& Twomey (1988), el entorno familiar como factor interno condiciona a los diferentes miembros en actividades empresariales. Otros estudios muestran que los emprendedores cuyos padres eran propietarios de pequeñas empresas a las que le dedicaban tiempo completo muestran más altas preferencias por el autoempleo y están más inclinados a desarrollar una idea empresarial, reflejando la menor preferencia por ser empleado en una gran empresa. Autores como Koellinger \& Minniti (2006) han mostrado que el entorno es un componente relevante en la decisión de una persona al momento de crear su empresa.

Liñán y Chen (2009) consideran que las reglas intangibles miden la presión que percibe el estudiante de la opinión de su entorno inmediato sobre el hecho de emprender una nueva idea o iniciativa de negocios. Es decir, estas reglas ejercen influencia sobre la persona, presionando a la hora de optar o no por el emprendimiento de una nueva iniciativa. La presión es realizada por la valoración que le otorga la familia, los amigos y los colegas a la aventura de emprender.

A pesar de lo anterior, se han encontrado estudios que no han podido establecer una relación directa entre estas normas y la intención emprendedora (Krueger, Reilly \& Carsrud, 2000); mientras que otras investigaciones demuestran que sí existe tal relación y que esta es positiva (Kolvereid $\&$ Isaksen, 2006).

Tomando en consideración los aspectos anteriores, se propone la siguiente hipótesis de trabajo:

- Hipótesis 6. Hay relación significativa entre las normas subjetivas que representan el entorno del estudiante (familia, compañeros de estudio y profesores) y la intención emprendedora del estudiante universitario. 


\subsection{Actitud emprendedora}

Peter Drucker (1985), uno de los autores más importantes en el tema, define al entrepreneur como aquel empresario que es innovador. El autor aclara la común confusión de creer que cualquier negocio pequeño y nuevo es un emprendimiento y que quien lo lleva a cabo es un emprendedor. Destaca que, aunque quien abra un pequeño negocio corra riesgos, eso no quiere decir que sea innovador y represente un emprendimiento. Además, afirma que el ser emprendedor no es un rasgo del carácter, sino una conducta, negando que esto sea producto de su personalidad.

\section{Metodología de la investigación}

El presente trabajo trata sobre la influencia del capital social y de las redes sociales en la actitud emprendedora de estudiantes de cinco universidades de la República Dominicana: INTEC, PUCMM, UNAPEC, UNIBE y UNPHU. En este apartado desarrollaremos la metodología de la investigación.

\subsection{Obtención de la muestra y recolección de datos}

El análisis se basa en fuentes de información primaria, con las bases de datos del registro de los estudiantes que han emprendido una iniciativa o algunos proyectos innovadores. La población de la investigación fue escogida de la lista de estudiantes que han tenido iniciativas emprendedoras. Esta lista fue distribuida para cada responsable de los centros de emprendimientos de las universidades seleccionadas. La población consultada representa un total de 92 estudiantes emprendedores que pertenecen a diferentes universidades ubicadas en Santo Domingo, República Dominicana. La distribución de la población por universidades se presenta en la siguiente tabla:
Tabla 1. Distribución total de estudiantes emprendedores según la universidad

\begin{tabular}{lrrrr}
\hline UNIVERSIDAD & $\begin{array}{c}\text { Frecuen- } \\
\text { cia }\end{array}$ & $\begin{array}{c}\text { Porcen- } \\
\text { taje }\end{array}$ & $\begin{array}{c}\text { Porcentaje } \\
\text { válido }\end{array}$ & $\begin{array}{r}\text { Porcentaje } \\
\text { acumulado }\end{array}$ \\
\hline UNAPEC & 27 & 29.3 & 29.3 & 100.0 \\
INTEC & 21 & 22.8 & 22.8 & 100.0 \\
PUCMM & 16 & 17.4 & 17.4 & 6.3 \\
UNIBE & 15 & 16.3 & 16.3 & 13.3 \\
UNPHU & 13 & 14.1 & 14.1 & 92.3 \\
\hline
\end{tabular}

Fuente: elaboración propia.

Esta investigación ha adoptado una estrategia de muestreo no probabilístico mediante el uso de una técnica de muestreo intencional que permitió al investigador seleccionar a los participantes del estudio. El método de muestreo intencional se establece con el fin de seleccionar los casos que se adapten mejor a las interrogantes y a los objetivos de la investigación (Saunders et al., 2003). El estudio es de tipo descriptivo. El propósito de la investigación descriptiva es producir una representación exacta de las personas, los eventos o la situación, lo que significa que se enfoca en hechos presentando unas características estudiadas (Saunders et al., 2006).

\subsection{Medición de variables}

La actitud emprendedora está basada en una predisposición a un comportamiento emprendedor que implica características del ser humano y del medio en el que opera. A los efectos de este estudio, vamos a definir la actitud emprendedora como la decisión de crear negocios, innovar y ańadir valor a los proyectos, procesos y productos en las organizaciones.

Para medir nuestra variable dependiente se tomaron en consideración las siguientes variables categóricas: claridad en la creación de empresas, preferencia por ser dueño de empresas y participación en grupos exclusivos de emprendedores. 
Estas categorías expresan una condición de deseabilidad para iniciar un nuevo emprendimiento (López \& García, 2011). La deseabilidad es un elemento de valor y se define como la motivación para complacer que se concreta en la aceptación y/o valoración de la opinión de los otros significativos (Morales, 1999). Así, "las intenciones conductuales (a las que se refiere la Teoría de la Acción Planificada) predicen mejor la conducta cuando van acompañadas por intenciones de implementación que indican dónde y cuándo hay que realizar la conducta" (Morales, 1999, p. 212).

\subsubsection{Variables independientes}

Las variables sociodemográficas referidas para los participantes en el estudio se incluyen por la importancia que tienen para la caracterización de la actitud emprendedora. En ese sentido, se ha identificado las siguientes:

- Edad, considerando que la literatura establece que las personas con mayor probabilidad de emprender un negocio son aquellas cuyas edades están comprendidas entre 25 y 34 años.

- Género, pues los estudios establecen diferencias en la actitud emprendedora de hombres y mujeres, demostrando una mayor preferencia de los varones hacia la creación de empresas en comparación con las mujeres.

- Nivel educativo, definida para los fines de este estudio por los niveles de grado y postgrado que cursan los estudiantes y el avance en la carrera en que están: bajo (un año o menos de cursado); medio (más de un año y menos de tres); alto (de tres ańos en adelante). Algunos actores consideran que el nivel educativo incide de diferentes maneras en la actitud emprendedora de las personas.

Las variables psicológicas, referidas a la propensión al riesgo y la necesidad de logros, se sustentan sobre el hecho de que los seres humanos en la búsqueda incesante por alcanzar el éxito tienen la capacidad de enfrentarse a situaciones contingentes que son riesgosas y, por lo tanto, esto los hace enfrentarlas de manera particular.

Las variables sociológicas o relacionales consideradas en esta investigación fueron el capital social, las redes sociales y el entorno familiar. Según Graña (2002), la actividad de creación de una empresa involucra un conjunto de factores culturales, económicos, sociológicos y psicológicos que adquieren características propias, a través de la vida y las experiencias de las personas en su proceso de socialización por lograr sus metas.

\section{Análisis de resultados}

Con el objetivo de alcanzar una mayor comprensión de los datos recopilados en la encuesta, así como de las características y los rasgos que definen el comportamiento emprendedor de la población objeto de estudio, se han considerado procesos estadísticos mediante el análisis de comparación de medias, prueba $\mathrm{T}$ para muestras independientes, ANOVA de un factor y correlaciones bivariadas de Spearman en variables categóricas.

\subsection{Factores sociodemográficos}

Edad: examinando la edad del emprendedor universitario con relación a la actitud emprendedora, como se presenta en las tablas 2, 3 y 4 , se evidencia que no hay diferencias significativas entre grupos de edades y actitud emprendedora; de igual manera, tampoco existen diferencias importantes entre esos grupos de edades y la actitud emprendedora por universidad.

Se comprobó con la prueba de Levene, para la igualdad de varianzas y de comparación múltiple post host DMS, que la Sig. intergrupal es 0.529; mientras que en la prueba de Levene para muestras independientes es de 0.916. Lo que sugiere rechazar 
la hipótesis de que existe una relación significativa entre la edad y la actitud emprendedora, tanto para los estudiantes considerados en el estudio como para los grupos de universidades.
Tabla 2. Análisis de varianza de la actitud emprendedora y los diferentes rangos de edades

\begin{tabular}{lccccc}
\multicolumn{1}{l}{ ANOVA } \\
\hline \multicolumn{1}{l}{ Actitud Emprendedora } \\
\hline & $\begin{array}{c}\text { Suma de } \\
\text { cuadrados }\end{array}$ & $\mathrm{gl}$ & $\begin{array}{c}\text { Media } \\
\text { cuadrática }\end{array}$ & F & Sig. \\
Inter-grupos & .749 & 3 & .250 & .745 & .529 \\
Intra-grupos & 21.121 & 63 & .335 & & \\
Total & 21.871 & 66 & & & \\
\hline
\end{tabular}

Fuente: elaboración propia.

\section{Tabla 3. Comparaciones múltiples entre diferentes rangos de edades}

Comparaciones múltiples. Pruebas post hoc

Actitud Emprendedora DMS

\begin{tabular}{|c|c|c|c|c|c|c|}
\hline \multirow[b]{2}{*}{ (I) Edad } & \multirow[b]{2}{*}{ (J) Edad } & \multirow[b]{2}{*}{$\begin{array}{c}\text { Diferencia de } \\
\text { medias (I-J) }\end{array}$} & \multirow[b]{2}{*}{ Error típico } & \multirow[b]{2}{*}{ Sig. } & \multicolumn{2}{|c|}{ Intervalo de confianza al 95\% } \\
\hline & & & & & Límite inferior & $\begin{array}{l}\text { Límite } \\
\text { superior }\end{array}$ \\
\hline \multirow[t]{3}{*}{ Menos de 20 años } & 20-24 años & .31566 & .21774 & .152 & -.1195 & .7508 \\
\hline & 25-30 años & .28118 & .23069 & .227 & -.1798 & .7422 \\
\hline & 31-40 años & .17063 & .34795 & .626 & -.5247 & .8660 \\
\hline \multirow[t]{3}{*}{ 20-24 años } & Menos de 20 años & -.31566 & .21774 & .152 & -.7508 & .1195 \\
\hline & 25-30 años & -.03448 & .16163 & .832 & -.3575 & .2885 \\
\hline & 31-40 años & -.14502 & .30655 & .638 & -.7576 & .4676 \\
\hline \multirow[t]{3}{*}{ 25-30 años } & Menos de 20 años & -.28118 & .23069 & .227 & -.7422 & .1798 \\
\hline & 20-24 años & .03448 & .16163 & .832 & -.2885 & .3575 \\
\hline & 31-40 años & -.11054 & .31588 & .728 & -.7418 & .5207 \\
\hline \multirow[t]{3}{*}{ 31-40 años } & Menos de 20 años & -.17063 & .34795 & .626 & -.8660 & .5247 \\
\hline & 20-24 años & .14502 & .30655 & .638 & -.4676 & .7576 \\
\hline & 25-30 años & .11054 & .31588 & .728 & -.5207 & .7418 \\
\hline
\end{tabular}

Fuente: elaboración propia. 
Tabla 4. Estadística del procedimiento Prueba $T$ para muestras independientes entre diferentes rangos de edades con respecto a la actitud emprendedora

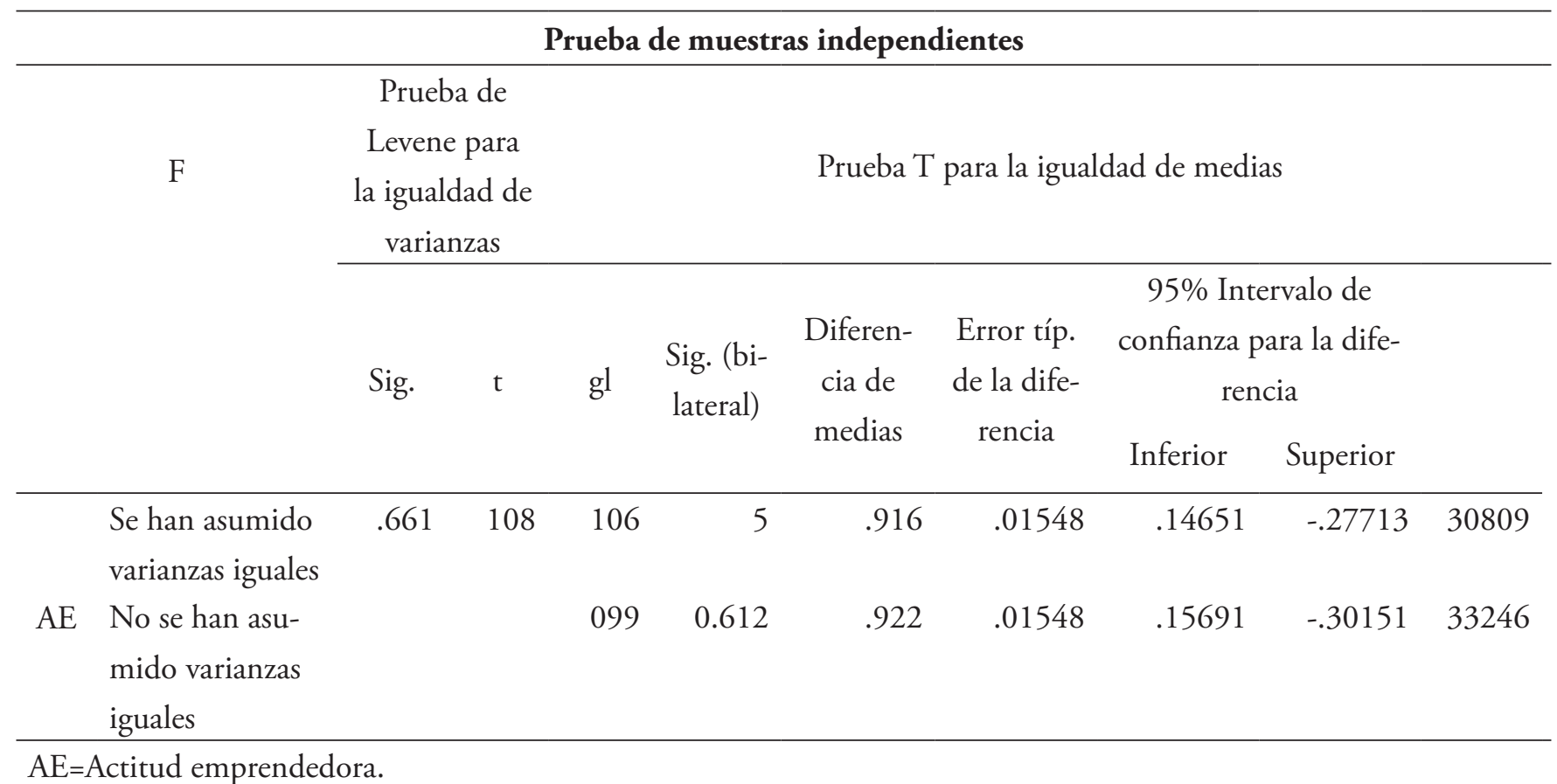

Fuente: elaboración propia.

Tabla 5. Prueba de muestras independientes $(t)$ entre actitud emprendedora y género

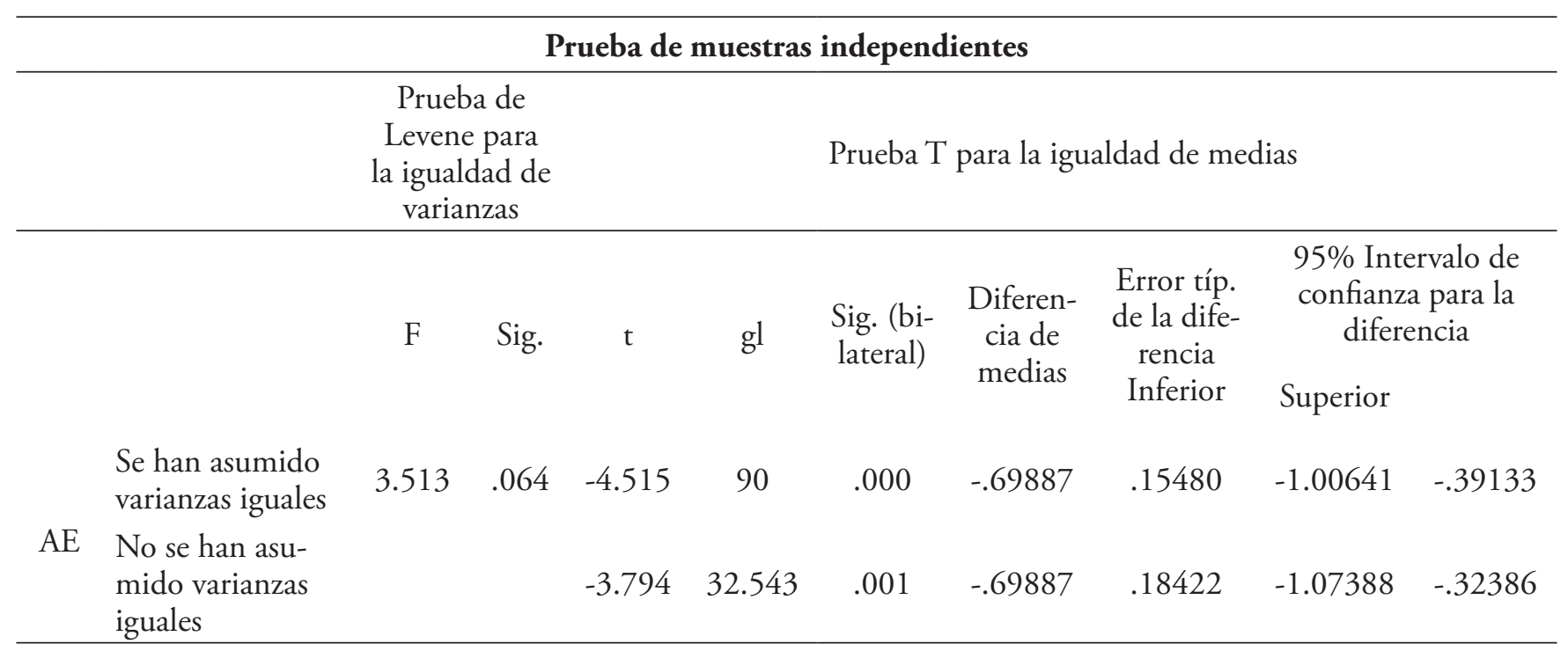

$\mathrm{AE}=$ Actitud emprendedora. 
Al observar la tabla 5 sobre prueba de muestras independientes $(t)$ entre actitud emprendedora $y$ género, se evidencia que no hay diferencias significativas entre hombres y mujeres con respecto a su actitud emprendedora, tanto de manera independiente como inter-universitario. En la misma tabla, a través de la Prueba de Levene para la igualdad de varianzas, se muestra que el estadio asociado a esta prueba es de 0.064 , superior a 0.05 , por lo que se rechaza la hipótesis nula $2 \mathrm{~b}$, lo que indica que no hay diferencia significativa entre la actitud emprendedora de estudiantes del género femenino y masculino de las universidades participantes en el estudio, por lo que se asume que las filas de las varianzas son iguales.

\section{Tabla 6. Media comparada de la actitud empren-} dedora según el nivel educativo

\begin{tabular}{llllll}
\hline & $\begin{array}{l}\text { Nivel del } \\
\text { programa } \\
\text { que estudia }\end{array}$ & $\mathrm{N}$ & Media & $\begin{array}{l}\text { Des- } \\
\text { viación }\end{array}$ & $\begin{array}{l}\text { Error típ. de } \\
\text { la media }\end{array}$ \\
\hline Actitud & Grado & 85 & 3.5662 & .73771 & .08002 \\
$\begin{array}{l}\text { Empren- } \\
\text { dedora }\end{array}$ & Postgrado & 7 & 3.6293 & .63500 & .24001 \\
\hline
\end{tabular}

En el caso de la comparación de las medias de los estudiantes que cursan el nivel de grado con los estudiantes que cursan el nivel de postgrado, se observa en la tabla 6 que existe una diferencia muy mínima (0.0631) en la media de los diferentes grupos de estudiantes de grado (3.5662) y postgrado (3.6293), con relación a su actitud emprendedora, siendo la media de postgrado un poco superior; sin embargo, esta diferencia no es significativa, como se evidencia en la tabla 7 de la prueba t de muestras independientes, por lo que se rechaza la hipótesis nula que establece que los estudiantes de nivel de grado tienen menor actitud emprendedora que los estudiantes del nivel de postgrado.

\section{Tabla 7. Prueba de muestras independientes $(t)$ entre actitud emprendedora y nivel educativo}

\begin{tabular}{|c|c|c|c|c|c|c|c|c|c|c|}
\hline \multicolumn{11}{|c|}{ Prueba de muestras independientes } \\
\hline & \multirow[t]{3}{*}{$\mathrm{F}$} & $\begin{array}{r}\text { Prue } \\
\text { Lever } \\
\text { la igua } \\
\text { vari }\end{array}$ & $\begin{array}{l}\text { de } \\
\text { para } \\
\text { ad de } \\
\text { zas }\end{array}$ & \multicolumn{7}{|c|}{ Prueba t para la igualdad de medias } \\
\hline & & \multirow[t]{2}{*}{ Sig. } & \multirow[t]{2}{*}{$\mathrm{t}$} & \multirow[t]{2}{*}{$\mathrm{gl}$} & \multirow{2}{*}{$\begin{array}{l}\text { Sig. (bi- } \\
\text { lateral) }\end{array}$} & \multirow{2}{*}{$\begin{array}{l}\text { Diferen- } \\
\text { cia de } \\
\text { medias }\end{array}$} & \multirow{2}{*}{$\begin{array}{l}\text { Error típ. } \\
\text { de la dife- } \\
\text { rencia }\end{array}$} & \multicolumn{2}{|c|}{$\begin{array}{c}\text { 95\% Intervalo de } \\
\text { confianza para la } \\
\text { diferencia }\end{array}$} & \\
\hline & & & & & & & & Inferior & Superior & \\
\hline & $\begin{array}{l}\text { Se han asumido } \\
\text { varianzas iguales }\end{array}$ & 061 & 806 & .219 & 90 & .827 & -.06301 & .28757 & -.63430 & .50829 \\
\hline $\mathrm{AE}$ & $\begin{array}{l}\text { No se han asu- } \\
\text { mido varianzas } \\
\text { iguales }\end{array}$ & & & .249 & .401 & .810 & -.06301 & .25299 & -.65473 & .52872 \\
\hline
\end{tabular}

$\mathrm{AE}=$ Actitud emprendedora. 
Cuando se evaluó la diferencia por comparación de medias entre la actitud emprendedora y el nivel de avance en la carrera, se observó que el nivel de significancia es superior a 0.005 , por lo tanto, se acepta la hipótesis nula $1 \mathrm{~d}$ de que no hay diferencias significativas en la actitud emprendedora de estudiantes con diferentes niveles de avance en la carrera que estudian.

\subsection{Factores psicológicos}

De acuerdo con la tabla 8, sobre el coeficiente de correlación de Spearman (rho), se observa una relación positiva de 0.303 y 0.320 y altamente significativa a nivel de 0.01 , entre la propensión a asumir riesgos y la necesidad de logros y la actitud emprendedora, respectivamente, confirmando las hipótesis nulas $\mathrm{H} 2 \mathrm{a}$ y $\mathrm{H} 2 \mathrm{~b}$ que suponen que la propensión al riesgo tiene una relación positiva con la actitud emprendedora de los estudiantes universitarios y que la necesidad de logros influye positivamente en la actitud emprendedora de los mismos.

\subsection{Factores sociológicos o relacionales}

\subsubsection{Capital social}

Como se muestra en la figura 1, la media es superior al punto medio de la escala, tanto en el capital social, que promedió 3.4790 , como en cada una de las dimensiones que lo componen. La reciprocidad fue el factor más valorado de las diferentes dimensiones del capital social. Alcanzó un valor promedio de 3.8524, en una escala de 1 a 5 donde $1=$ bajo, 3 = medio, 5 = alto. Lo que significa que el $77.048 \%$ de los participantes del estudio se perciben son recíproco en su comportamiento.

\section{Figura 1. Valor promedio de las dimensiones de capital}

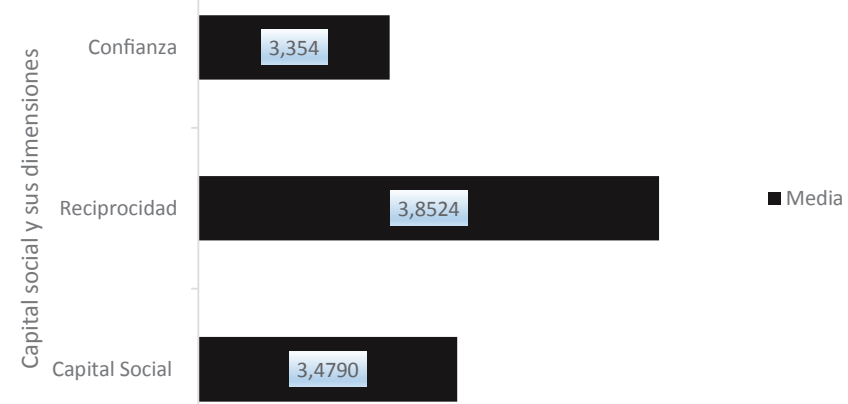

Tabla 8. Coeficiente de correlación de Spearman entre actitud emprendedora y propensión a tomar riesgos y la actitud de logros

\begin{tabular}{|c|c|c|c|c|}
\hline VARIABLES & $\begin{array}{l}\text { Rho de Spear- } \\
\text { man }\end{array}$ & $\begin{array}{c}\text { Actitud empren- } \\
\text { dedora }\end{array}$ & Riesgos & Logros \\
\hline Actitud emprendedora & $\begin{array}{l}\text { Correlation coe- } \\
\text { fficient } \\
\text { Sig. (bilateral) }\end{array}$ & $\begin{array}{l}1.000 \\
0.000\end{array}$ & $\begin{array}{c}0.303^{* *} \\
0.006\end{array}$ & $\begin{array}{l}0.320^{* *} \\
0.002\end{array}$ \\
\hline Riesgos & $\begin{array}{l}\text { Correlation coe- } \\
\text { fficient } \\
\text { Sig. (bilateral) }\end{array}$ & $\begin{array}{l}0.303^{* *} \\
0.006\end{array}$ & $\begin{array}{l}1.000 \\
0.000\end{array}$ & $\begin{array}{c}0.260^{*} \\
0.20\end{array}$ \\
\hline Logros & $\begin{array}{l}\text { Correlation coe- } \\
\text { fficient } \\
\text { Sig. (bilateral) }\end{array}$ & $\begin{array}{l}0.320^{* *} \\
0.002\end{array}$ & $\begin{array}{c}0.260^{*} \\
0.20\end{array}$ & $\begin{array}{l}1.000 \\
0.000\end{array}$ \\
\hline
\end{tabular}

**. La correlación es significativa al nivel 0,01 (bilateral).

*. La correlación es significativa al nivel 0,05 (bilateral). 
Según la figura 1, el factor confianza alcanzó una media de (3.35). Corroborando con estos resultados, Saniz y Jiménex (2008) opinan que los valores de relación que se expresan en las prácticas informales de comportamiento son derivados de la reciprocidad y la confianza que se da entre las personas.

Tabla 9. Media del capital social según universidades donde estudian

\begin{tabular}{cccc} 
& \multicolumn{3}{c}{ Capital social } \\
Universidad en & & & \\
\cline { 2 - 4 } la que estudia & $\mathrm{N}$ & Media & Desv. típ. \\
& & & \\
\hline UNAPEC & 26 & 3.4250 & .53058 \\
INTEC & 18 & 3.6111 & .44108 \\
PUCMM & 14 & 3.4647 & .40442 \\
UNIBE & 13 & 3.5406 & .36964 \\
UNPHU & 11 & 3.3364 & .77495 \\
\hline
\end{tabular}

Fuente: elaboración propia

Analizando la tabla 9, en el caso del capital social, todos los emprendedores de las universidades participantes superan la media superior de la escala de 1 a 5 , donde 1 = bajo, 3 = medio, 5 = alto. Los resultados de las medias de las diferentes universidades oscilan en valores comprendidos entre $3.6111 \mathrm{y}$ 3.3364 .
Tabla 10. Coeficiente de correlación de Spearman entre capital social y actitud emprendedora

\begin{tabular}{|c|c|c|c|c|}
\hline & & & $\begin{array}{l}\text { Social } \\
\text { capital }\end{array}$ & $\begin{array}{l}\text { Actitud } \\
\text { empren- } \\
\text { dedora }\end{array}$ \\
\hline \multirow{6}{*}{$\begin{array}{l}\text { Rho de } \\
\text { Spear- } \\
\text { man }\end{array}$} & \multirow[t]{3}{*}{$\begin{array}{l}\text { Social } \\
\text { capital }\end{array}$} & $\begin{array}{l}\text { Correlation } \\
\text { coefficient }\end{array}$ & 1.000 & $.543^{* *}$ \\
\hline & & $\begin{array}{l}\text { Sig. (bilate- } \\
\text { ral) }\end{array}$ & $\cdot$ & .000 \\
\hline & & $\mathrm{N}$ & 82 & 82 \\
\hline & \multirow{3}{*}{$\begin{array}{l}\text { Actitud } \\
\text { empren- } \\
\text { dedora }\end{array}$} & $\begin{array}{l}\text { Correlation } \\
\text { coefficient }\end{array}$ & $.543^{* *}$ & 1.000 \\
\hline & & $\begin{array}{l}\text { Sig. (bilate- } \\
\text { ral) }\end{array}$ & .000 & \\
\hline & & $\mathrm{N}$ & 82 & 82 \\
\hline \multicolumn{5}{|c|}{$\begin{array}{l}\text { **. Correlation is significant at the } 0.01 \text { level (bilateral). } \\
\text { La tabla } 10 \text { permite apreciar la correlación de } \\
\text { Spearman, donde se muestra que el capital social } \\
\text { tiene una fuerte correlación positiva de } 0.543 \text {, con } \\
\text { una significancia de } .000 \text {, a un nivel de confianza } \\
\text { de } 0.01 \text { con la actitud emprendedora. Por lo que se } \\
\text { acepta la hipótesis nula H3 que establece que el de- } \\
\text { sarrollo del capital social se relaciona positivamente } \\
\text { con el desarrollo de la actitud emprendedora de los } \\
\text { estudiantes. }\end{array}$} \\
\hline
\end{tabular}

Tabla 11. Análisis de varianza del capital social según universidades

ANOVA

Capital social

\begin{tabular}{lrrrrr}
\hline & $\begin{array}{l}\text { Suma de } \\
\text { cuadrados }\end{array}$ & gl & $\begin{array}{l}\text { Media } \\
\text { cuadrá- } \\
\text { tica }\end{array}$ & F & Sig. \\
\hline Inter-grupos & .666 & 4 & .167 & .67 & .637 \\
Intra-grupos & 20.117 & 77 & .261 & & \\
Total & 20.783 & 81 & & & \\
\hline
\end{tabular}

Fuente: elaboración propia. 
Tabla 12. Coeficiente de correlación Spearman y las variables relacionadas con las redes y la actitud emprendedora

\begin{tabular}{|c|c|c|c|c|}
\hline & & & $\begin{array}{l}\text { Actitud } \\
\text { empren- } \\
\text { dedora }\end{array}$ & $\begin{array}{l}\text { Redes } \\
\text { sociales }\end{array}$ \\
\hline \multirow{6}{*}{$\begin{array}{l}\text { Rho de } \\
\text { Spear- } \\
\text { man }\end{array}$} & $\begin{array}{l}\text { Actitud } \\
\text { em- }\end{array}$ & $\begin{array}{c}\text { Coeficiente de } \\
\text { correlación }\end{array}$ & 1.000 & $.474^{* *}$ \\
\hline & prende- & Sig. (bilateral) & . & .000 \\
\hline & dora & $\mathrm{N}$ & 67 & 67 \\
\hline & Redes & $\begin{array}{l}\text { Coeficiente de } \\
\text { correlación }\end{array}$ & $.474^{* *}$ & 1.000 \\
\hline & sociales & Sig. (bilateral) & .000 & . \\
\hline & & $\mathrm{N}$ & 67 & 67 \\
\hline
\end{tabular}

**. La correlación es significativa al nivel 0,01 (bilateral) Fuente: elaboración propia

Tabla 13. Coeficiente de correlación de Spearman sobre las variables relacionadas con el entorno del estudiante y la actitud emprendedora

\begin{tabular}{|c|c|c|c|c|}
\hline & & & $\begin{array}{r}\text { Actitud } \\
\text { empren- } \\
\text { dedora }\end{array}$ & $\begin{array}{c}\text { Entor- } \\
\text { no }\end{array}$ \\
\hline \multirow{9}{*}{$\begin{array}{l}\text { Rho de } \\
\text { Spear- } \\
\text { man }\end{array}$} & \multirow{4}{*}{$\begin{array}{l}\text { Actitud } \\
\text { empren- } \\
\text { dedora }\end{array}$} & Coeficiente & & $.220^{*}$ \\
\hline & & $\begin{array}{l}\text { de correla- } \\
\text { ción }\end{array}$ & 1.000 & \\
\hline & & $\begin{array}{l}\text { Sig. (bilate- } \\
\quad \text { ral) }\end{array}$ & $\cdot$ & .035 \\
\hline & & $\mathrm{N}$ & 92 & 92 \\
\hline & \multirow{5}{*}{ Entorno } & Coeficiente & $.220^{*}$ & \\
\hline & & de correla- & & 1.000 \\
\hline & & ción & & \\
\hline & & $\begin{array}{l}\text { Sig. (bilate- } \\
\text { ral) }\end{array}$ & .035 & $\cdot$ \\
\hline & & $\mathrm{N}$ & 92 & 92 \\
\hline
\end{tabular}

\footnotetext{
*. La correlación es significativa al nivel 0,05 (bilateral).
}

\subsubsection{Redes sociales}

La tabla 12 presenta el coeficiente de correlación de Spearman sobre las variables relacionadas con las redes y la actitud emprendedora, la cual muestra que existe una correlación significativa entre las variables relacionadas con las redes sociales y la actitud emprendedora de $0.474^{* *}$, lo que significa que hay evidencia estadística para aceptar la hipótesis nula $\mathrm{H} 5$ de que hay relación significativa entre el uso de las redes sociales y el desarrollo de la actitud emprendedora en estudiantes universitarios.

\subsubsection{Entorno familiar}

La tabla 13 sobre el coeficiente de correlación de Spearman, acerca de las variables relacionadas con el entorno del estudiante y la actitud emprendedora, muestra que existe correlación significativa entre las variables relacionadas con el entorno y la actitud emprendedora, con un valor de $0.020^{*}$ y una significancia de 0.035 , por lo que se acepta la hipótesis nula $\mathrm{H} 6$ de que hay relación positiva entre el entorno y el desarrollo de la actitud emprendedora en estudiantes.

\section{Conclusiones}

En este trabajo se han analizado los factores explicativos de la actitud emprendedora en estudiantes de cinco universidades de la República Dominicana, desde la perspectiva de las características sociodemográficas, los factores psicológicos y los factores sociológicos o relacionales del emprendedor universitario, focalizando el estudio en universidades que han establecido centros de emprendedurismo con estudiantes.

Aunque existe una amplia literatura sobre la actitud emprendedora y los factores que la explican, esta investigación esencialmente se ha focalizado en establecer la relación de los factores demográficos: edad, género y nivel educativo, que según estudios contraponen resultados; de igual manera, se ha presentado la relación de los factores psicológicos 
y sociológicos como el capital social, las redes y el entorno familiar como determinantes de la actitud emprendedora.

En República Dominicana existen muy pocas investigaciones que profundicen sobre el impacto de las dimensiones del capital social y la influencia del uso de las redes sociales en el comportamiento de los emprendedores. Esto es relevante dada la necesidad de fomentar la actitud emprendedora en jóvenes universitarios, a través de procesos que viabilicen el desarrollo de iniciativas colectivas, necesarias para potencializar la actitud emprendedora de las personas.

Podemos concluir que los factores explicativos de la actitud emprendedora están vinculados a los componentes psicológicos y sociológicos. Los factores sociodemográficos como el género, la edad y el nivel educativo no explican este tipo de comportamiento en la población estudiada. El análisis de 92 casos recopilados en la encuesta aplicada en 2016 arroja datos empíricos que permiten esta conclusión y, por tanto, se aceptan algunas de las hipótesis planteadas en la investigación. Hay relación significativa entre la necesidad de logros y la propensión al riesgo con la actitud emprendedora; también se confirma que existen relaciones significativas entre el capital social y dicha actitud.

Las redes sociales como medio para compartir y transferir conocimiento y experiencia, así como para fomentar el trabajo colaborativo, explican favorablemente la actitud emprendedora, al igual que el entorno familiar. Aquellos estudiantes que cuentan con familiares y amigos cercanos que tienen empresas presentan mayor desarrollo en su actitud emprendedora.

Los resultados de esta investigación tienen importantes implicaciones y pueden dar pautas a las universidades para incentivar la actitud emprendedora en los estudiantes universitarios, fomentando la confianza, la reciprocidad y los proyectos colaborativos que impliquen retos y riesgos.
Las universidades, a través de sus mecanismos formales, deben invertir tiempo y esfuerzo para facilitar al estudiante emprendedor la inserción en redes de relacionados, de modo que les permita utilizar estas redes para acceder a recursos y capacidades colectivas, lo que por sí solo sería difícil de lograr.

Este estudio presenta la necesidad de fortalecer el desarrollo de pasantías intensivas para estudiantes con actitud emprendedora, a los fines de establecer mecanismos que favorezcan la articulación y coordinación de estos estudiantes con sectores y eslabones esenciales de la cadena productiva, relacionados con las iniciativas emprendedoras que desarrollan. Es conveniente ampliar y fortalecer los vínculos, pues es necesario alentar el capital social relacional entre los estudiantes emprendedores.

Es favorable que cuando un miembro del ecosistema emprendedor universitario o una red ocupen una posición central o un liderazgo adopten una orientación relacional con sus contactos directos, para de este modo mejorar el funcionamiento de todo el grupo. Este tipo de comportamientos relacionales son muy favorecidos por la cohesión interna de las redes, de manera que potencializan el desarrollo de la actitud emprendedora del estudiante.

Este estudio presenta varias limitaciones que sugieren vías de investigación futuras. En primer lugar, las propias limitaciones de las bases de datos, ya que cada universidad envió el cuestionario a sus emprendedores registrados, dificultando el seguimiento para incrementar el número de personas que respondieran el instrumento. Es importante establecer un registro único de emprendedores universitarios para dar seguimiento y apoyo a sus iniciativas.

Finalmente, entendemos que se precisa profundizar el análisis de los factores que explican la actitud emprendedora en estudiantes universitarios, pues se evidencia una minoría muy exclusiva de estudiantes que muestran este tipo de comportamiento. 


\section{Referencias}

Ajzen, I. (1991). The theory of planned behavior. Organizational behavior and human decision processes, 50, 179-211.

Astri, R. (2001). Capital social y reducción de la pobreza en América Latina y el Caribe. Chile: CEPAL.

Banco Mundial. (1999). Capital social en español. Recuperado de http://web.worldbank.org Y http:// www.cepal.org/publicaciones/xml/8/13428/panel1_sps31.pdf

Baron, R. A. (2000). The cognitive perspective: A valuable tool for answering entrepreneurship's basic "why" questions. Journal of Business Venturing, 19(2), 221-239.

Blanchflower, D. (2004). Self-employment: more may not be better. Swedish Economic Policy Review, 11(2), 15-74.

Bourdieu, P. (1986). The forms of capital. En J. G. Richardson (Ed.). Handbook of theory and research for the sociology of education. New York: Greenwood. Recuperado de https://faculty.georgetown.edu/irvinem/theory/Bourdieu-Forms-of-Capital.pdf

Cano, C.J., García, J. \& Gea, A. B. (2004). Actitudes emprendedoras en los estudiantes universitarios. En Roig, S.; Ribeiro, D.; Torcal, R.; De la Torre, A. \& Cerver, E. (2004). El emprendedor innovador y la creación de empresas de $I+D+I$. Servei de Publicacións Universitat de Valencia, cap. 9, 143-160.

Catalso, F., Herrera, F., Rojas, D. \& Rojas, T. (2012). La evaluación de la propensión al riesgo jes confiable el uso del "test de alerta"? [Estudio exploratorio en trabajadores de servicios a la minería]. Universidad Católica del Norte, Antofagasta, Chile.

Entrialgo, M.; Fernández, E. \& Vázquez, C. J. (1999). Las características del propietario/directivo de la PYME como factores determinantes de su comportamiento emprendedor. Boletin de Estudios Económicos, 54(168), 405-424.
Coleman, J. (1990). Foundations of social theory. Cambridge: Harvard University Press.

Coleman, J. S. (1998). Social Capital in the Creation of Human Capital. The American Journal of Sociology, 94, 95-120.

Durston, J. (2000). ¿Qué es el capital social comunitario? Recuperado de http://www.cepal.org/es/publicaciones/5969-que-es-el-capital-social-comunitario

Graña, F. (2002) Creación de empresas: factores asociados al éxito emprendedor en cinco ciudades argentinas. Recuperado de https://core.ac.uk/download/ pdf/11676820.pdf

Gibb, A. \& Ritchie J. (1982). Understanding the process of starting small businesses. European Small Business Journal, 1(1), 26-46. Sage Journal Recuperado de http://isb.sagepub.com/content/1/1/26.full.pdf

Johannisson, B. (2006). El desarrollo regional como red de contactos empresariales: de la elección racional a la autoorganización. Ekonomiaz, 62(2), 220-239.

Kolvereid, L. \& Isaksen, E. (2006). New business start-up and subsequent entry into selfemployment. Journal of Business Venturing, 21(6), 866885. Recuperado de http://www.sciencedirect. com/science/article/pii/S0883902605000686

Koellinger, P.; Minniti, M. \& Schade, C. (2007). I think I can, I think I can: overconfidence and entrepreneurial behavior. Journal of Economic Psychology, 28(4), 502-527. DOI: 10.1016/j. joep.2006.11.002 Syracuse University. EEUU.

Krueger, N. F., Reilly M. D. \& Carsrud, A. L. (2000). Competing Models of Entrepreneurial Intentions. Journal of Business Venturing, 15(5-6), 411-432. Recuperado de http:// www.sciencedirect.com/science/article/pii/ S0883902698000330

Levesque, M. \& Minniti, M. (2006). The effect of aging on entrepreneurial behavior.Journal of Business 
Venturing, 21(2), 177-194. DOI: 10.1016/j.jbusvent.2005.04.003 Syracuse University. EEUU.

Liñán, F. \& Chen, Y. (2009). Development and cross-cultural application of a specific instrument to measure entrepreneurial intentions. Entrepreneurship: Theory \& Practice, 33(3), 593-617. DOI: 10.1111/ j.1540-6520.2009.00318. Baylor University. Recuperado de http://onlinelibrary.wiley.com/ doi/10.1111/j.1540-6520.2009.00318.x/epdf?r3_ referer=wol\&tracking_action=preview_click\&show_ checkout=1\&purchase_referrer=www.google.com. do\&purchase_site_license=LICENSE_DENIED

López, J. \& García, J. (2011). Optimismo, pesimismo y realismo disposicional en emprendedores potenciales de base tecnológica. Psicothema, 23 (4), 611-616. Recuperado de http://www.psicothema. com/pdf/3930.pdf

Morales, F. (Coord.). (1999). Psicología Social. España: Mc Graw Hill.

Montesinos, V; Serrano, L.; Fernández, J. \& Pérez, F. (2005). La medición del capital social: una aproximación económica. Bilbao: Fundación BBVA.

Prahalad, C. \& Bettis, R. (1986). The dominant logic: A new linkage between diversity and performance. Strategic Management Journal, 7(6), 485-501. DOI: $10.1002 /$ smj.4250070602. Recuperado de http://hdl.handle.net/2027.42/106722

Putnam, R. (1993). Making democracy work: Civic tradition in modern Italy. Princeton: University Press of Princeton.

Putnam, R. (1993). The Prosperous Community: Social capital and public life. The American Prospect, 13. Disponible en http://epn.org/prospect/13/13putn.html.

Putman, R. (1995). Tuning in, Tuning out: The strange disappearance of social capital in America. Political Science and Politics, 664-683 Recuperado de http://www.jstor.org/stable/420517
Putnam, R. D. (2002). Bowling alone: The collapse of America's social capital. New York: Simon and Shuster. Recuperado de http://www.simonandschuster.com/books/Bowling-Alone/ Robert-D-Putnam/9780743203043/browse_inside

Rangel, S. (2008). Capital social: una revisión del concepto. Bogotá: Universidad Santo Thomas.

Robbins, S. \& Judge, T. (2011). Organizational behavior. New Jersey: Pearson Prentice Hall.

Robbins, S. (2011). Comportamiento organizacional, teoría y práctica. (3ra ed.). Editorial México: Prentice Hall Hispanoamericana S.A.

Saunders, M, Lewis, P. \& Thomhill, A. (2003). Research Methods for Business. México: Pearson, Cengage Learning.

Scout, M. G. \& Twomey, D. F. (1988). The longterm supply of entrepreneurs: Students career aspirations in relation to entrepreneurship. Journal of small business management, 26(4), 5-13. (Editado por George T. Solomon).

Veciana, J.; Aponte, M. \& Urbano, D. (2005). University student's attitudes towards entrepreneurship: A two countries comparison. International Entrepreneurship and Management Journal, 1, 165-18.

\section{Datos de filiación}

José Feliz Marrero, doctorado en Business Administración por Paris School of Business, máster of Science the Ohio State University (OSU); maestría en Ciencias Política por la Universidad de Salamanca; maestrías en Alta Gerencia y Maestría en Mercadeo (INTEC). Ha sido docente en diferentes programas de maestrías en el área de negocios del INTEC y otros centros de educación superior de República Dominicana. Actualmente se desempeña como vicerrector académico del INTEC. Sus publicaciones incluyen artículos y ponencias sobre temas de ciencias, tecnología e innovación. Correo electrónico: jose.feliz@intec.edu.do 